\title{
Application de la thermographie aéroportée à la prospection
} archéologique

Pierre Beaufrère, Michel Dabas, Jean Paul Décriaud, Alain Tabbagh, Charles Leva

\section{Citer ce document / Cite this document :}

Beaufrère Pierre, Dabas Michel, Décriaud Jean Paul, Tabbagh Alain, Leva Charles. Application de la thermographie aéroportée à la prospection archéologique. In: Revue archéologique de Picardie. Numéro spécial 17, 1999. Actes du colloque international d'archéologie aérienne Amiens, 15 - 18 octobre 1992. Hommage à Roger Agache pour 35 ans de prospections aériennes dans le Nord de la France. pp. 289-293;

doi : 10.3406/pica.1999.2115

http://www.persee.fr/doc/pica_1272-6117_1999_hos_17_1_2115

Document généré le 20/04/2016 


\begin{abstract}
The ARIES radiometer, built in 1975 by the LMD - CNRS, enables us to numerically acquire images both in the visible spectrum, in the thermal infrared region (10.5-12.5 um) and in the near IR region (0.5- $1.1 \mathrm{um}$ ). Experiments during the last decade have proved the importance of these data for regional archaeology both for the detection of archaeological remains and for the knowledge brought by micro topographic features significant of the landscape history. Anew software has been designed in 1991 in the Centre de Recherches Géophysiques of Garchy for downloading this information on a PC, visualize and process it interactively on a high definition screen. This software is portable. Data are stored on a magnetic tape or an optical disk. Color graphic output is done after palette selection and different numerical computations (geometrical corrections for the pitchroll-anamorphosis deformation, contrast enhancement and classification). This software has been developped for editing the images just a few hours after the flight. The information brought by the joint use of both channels is important as shown by the examples of a recent flight in Beauce. The choice of the time of flight is also very important and is the result of the monitoring, several weeks before the flight, of the temperature flux in the ground, through the existing meteorological network.
\end{abstract}

\title{
Résumé
}

Grâce à l'utilisation d'un radiomètre ARIES (mis au point en 1975 au LMD - CNRS) il nous a été possible d'acquérir numériquement des images à la fois dans le canal thermique (10,5-12,5 um) dans le spectre visible et dans le proche IR (0,5-1,1/1 um). Les expériences de ces dernières années ont montré tout l'intérêt de ces données en prospection archéologique régionale tant pour la détection des vestiges enfouis que pour la reconnaissance des micro-reliefs révélateurs de structures significatives de l'histoire du paysage. Un nouvel ensemble logiciel a été développé en 1991 au Centre de Recherches géophysiques de Garchy pour transférer l'information graphique sur un PC, la visualiser et la traiter de façon interactive sur un écran graphique. Cet ensemble est portable. Les informations sont stockées sur bandes magnétiques et disques optiques. Des sorties graphiques en couleurs sont effectuées après avoir choisi des palettes de couleur et les traitements numériques sur l'image (corrections géométriques visant à éliminer l'effet du roulis et du tangage, anamorphose, augmentation de contraste, sélection de classes). Cet ensemble a été développé afin de pouvoir éditer les résultats quelques heures après le vol même. L'information apportée par l'utilisation conjointe des canaux visibles et thermiques est importante comme le montreront les exemples des prospections effectuées en Beauce. Le choix de la période de vol est tout aussi important et résulte d'un suivi, pendant plusieurs semaines avant le vol prévu, du flux thermique dans le sol, à partir d'un réseau de stations météorologiques existantes. 


\title{
APPLICATION DE LA THERMOGRAPHIE AÉROPORTÉE À LA PROSPECTION ARCHÉOLOGIQUE
}

\author{
Pierre BEAUFRĖRE, Michel DABAS, Jean-Paul DÉCRIAUD (†), Alain TABBAGH *
}

\begin{abstract}
Résumé
Grâce à l'utilisation d'un radiomètre ARIES (mis au point en 1975 au LMD - CNRS) il nous a été possible d'acquérir numériquement des images à la fois dans le canal thermique $(10,5-12,5 \mu \mathrm{m})$ dans le spectre visible et dans le proche IR $(0,5-1,1 / 1 \mu \mathrm{m})$. Les expériences de ces dernières années ont montré tout l'intérêt de ces données en prospection archéologique régionale tant pour la détection des vestiges enfouis que pour la reconnaissance des micro-reliefs révélateurs de structures significatives de l'histoire du paysage. Un nouvel ensemble logiciel a été développé en 1991 au Centre de Recherches géophysiques de Garchy pour transférer l'information graphique sur un PC, la visualiser et la traiter de façon interactive sur un écran graphique. Cet ensemble est portable. Les informations sont stockées sur bandes magnétiques et disques optiques. Des sorties graphiques en couleurs sont effectuées après avoir choisi des palettes de couleur et les traitements numériques sur l'image (corrections géométriques visant à éliminer $l^{\prime}$ effet du roulis et du tangage, anamorphose, augmentation de contraste, sélection de classes). Cet ensemble a été développé afin de pouvoir éditer les résultats quelques heures après le vol même. L'information apportée par l'utilisation conjointe des canaux visibles et thermiques est importante comme le montreront les exemples des prospections effectuées en Beauce. Le choix de la période de vol est tout aussi important et résulte d'un suivi, pendant plusieurs semaines avant le vol prévu, du flux thermique dans le sol, à partir d'un réseau de stations météorologiques existantes.
\end{abstract}

\section{Abstract}

The ARIES radiometer, built in 1975 by the LMD - CNRS, enables us to numerically acquire images both in the visible spectrum, in the thermal infrared region $(10.5-12.5 \mu \mathrm{m})$ and in the near IR region $(0.5-$ $1.1 \mu \mathrm{m})$. Experiments during the last decade have proved the importance of these data for regional archaeology both for the detection of archaeological remains and for the knowledge brought by micro topographic features significant of the landscape history. A new software has been designed in 1991 in the Centre de Recherches Géophysiques of Garchy for downloading this information on a PC, visualize and process it interactively on a high definition screen. This software is portable. Data are stored on a magnetic tape or an optical disk. Color graphic output is done after palette selection and different numerical computations (geometrical corrections for the pitchroll-anamorphosis deformation, contrast enhancement and classification). This software has been developped for editing the images just a few hours after the flight. The information brought by the joint use of both channels is important as shown by the examples of a recent flight in Beauce. The choice of the time of flight is also very important and is the result of the monitoring, several weeks before the flight, of the temperature flux in the ground, through the existing meteorological network.

Le prospecteur aérien enregistre sur son émulsion photographique une partie du rayonnement réfléchi ou émis par la terre : la partie du spectre dite " visible " à l'œil humain. Mais il existe aussi une autre partie du spectre qui peut apporter une information au prospecteur archéologue.

\footnotetext{
* CNRS Centre de Recherches géophysiques UMR 76-19

F - 58150 GARCHY
}

En effet, la surface du sol ne fait pas que réfléchir le rayonnement solaire. Comme tout corps, le sol émet un rayonnement du fait de sa température propre.

C'est ce rayonnement que nous mesurons dans la prospection thermique aéroportée.

Au-delà du rayonnement visible $(0,4$ à $0,8 \mu \mathrm{m}$ de longueur d'onde), les prospecteurs utilisent déjà un rayonnement non visible, l'infrarouge proche 
(entre 0,8 et $1,1 \mu \mathrm{m}$ ), rayonnement qu'ils sont capables d'enregistrer grâce à des émulsions photographiques spéciales.

Malheureusement, on ne peut plus enregistrer aussi simplement les longueurs d'ondes plus élevées, longueurs d'onde d'autant plus intéressantes qu'elles portent une information bien différente de celle apportée par le spectre visible. L'enregistrement de ces grandes longueurs d'onde est difficile car on observe une diminution de l'énergie émise quand la longueur d'onde augmente. On a corrélativement un bruit qui augmente ainsi que des absorptions spécifiques du rayonnement par certaines molécules de l'atmosphère $\left(\mathrm{CO}_{2}\right.$, $\mathrm{H}_{2} \mathrm{O}$ ). Heureusement, il existe deux "fenêtres " où l'absorption est moindre : la première est située entre 3 et $5 \mu \mathrm{m}$. et la seconde entre 8 et $13 \mu \mathrm{m}$.

De plus, si l'on étudie la luminance (spectrale) d'un sol en fonction de la température et de la longueur d'onde (loi de PLANCK), on constate qu'un sol à une température de quelques degrés (conditions de vol le matin au printemps) possède un maximum de luminance vers $10 \mu \mathrm{m}$. qui correspond à l'une des deux fenêtres de moindre absorption de l'atmosphère. Nous allons donc enregistrer le rayonnement du sol à une longueur d'onde proche de $10 \mu \mathrm{m}$. Ce domaine s'appelle l'infrarouge thermique, car la mesure de luminance, après des calibrations complexes que nous n'aborderons pas ici, est une mesure de la température du sol.

Pourquoi donc mesurer la température du sol? Parce que celle-ci traduit la nature même du sol (au travers de deux variables) et ce jusqu'à une certaine profondeur. Mesurer des différences de température entre deux points revient donc à caractériser aussi bien deux sols d'origine pédologique différente, que deux sols dont les propriétés physiques diffèrent comme par exemple leur teneur en eau ou leur densité. L'application archéologique est donc importante comme l'a montré A. Tabbagh depuis 1976 (SCOLLAR et al., 1990). Mais de nombreuses autres communautés de chercheurs sont intéressées par ces mesures comme les urbanistes, les volcanologues ou les pédologues.

Dans le système d'acquisition des images thermiques, l'équivalent de la chambre de l'appareil photographique est le radiomètre. Celui que nous utilisons est capable d'enregistrer deux bandes de longueur d'onde simultanément. Cet appareil très précis, mis au point par le LMD (Laboratoire de Météorologie Dynamique, MONGE et SIRIOU, 1975) du CNRS comme prototype pour le radiomètre du satellite METEOSAT comprend un détecteur refroidi à l'azote liquide. C'est un appareil de mesure absolu car les calibrations sont effec- tuées en vol par rapport à des « corps noirs » dont on connaît exactement la température.

Associé au radiomètre, nous trouvons un gyroscope qui nous donne les angles de roulis et de tangage de l'avion ainsi que toute l'électronique d'acquisition et d'enregistrement du signal thermique. Le radiomètrc, commc un scanner d'ordinateur, balaye la scène sous l'avion grâce à un miroir tournant. On obtient donc une ligne de points. Le balayage dans le sens du profil est bien sûr obtenu par le déplacement de l'avion.

Toute l'information fournie par le radiomètre (fig. 1) est numérisée en temps réel, contrôlée par un opérateur sur un oscilloscope et stockée.

Les hauteurs de vol sont voisines de $600 \mathrm{~m}$. La vitesse de l'avion, un Pilatus, est de $40 \mathrm{~m} / \mathrm{s}$. La résolution au sol d'un point de l'image (pixel) est de $1 \mathrm{~m}$ dans les deux directions. En dix minutes de vol, nous balayons un couloir de $1 \mathrm{~km}$ de large et de $24 \mathrm{~km}$ de longueur, ce qui représente 25 mégaoctets d'information sur la bande. La résolution en température est de $0,05{ }^{\circ} \mathrm{C}$. Nous utilisons des filtres sur le radiomètre qui nous permettent d'enregistrer simultanément dans le domaine visible et IR thermique.

L'opérateur dans l'avion dispose aussi d'une caméra vidéo et d'un écran de visualisation pour le contrôle des zones survolées. L'équipage est ordinairement constitué d'un pilote, d'un copilote chargé de la navigation et du suivi des profils, d'un ou deux opérateurs pour le contrôle de l'acquisition (fig. 2).

Nous présentons en trois points l'originalité de notre système depuis les développements effectués cette année ( $c f . \mathrm{J}$. du CNRS n 33 ).

\section{LE TRAITEMENT}

Jusqu'à présent, l'obtention des résultats sur plan film nécessitait des délais importants : en effet le vol achevé, les bandes (en format PCM) contenant les données, leur calibration et les données de navigation étaient lues au centre de calcul de l'École Polytechnique pour obtenir une bande numérique classique. Ce traitement fini, nous faisions différents calculs sur l'image dans un autre centre de calcul (CIRCE à Orsay) et les images finales étaient finalement tirées sur plan-film au Rheinisches Landesmuseum à Bonn (Laboratoire de I. SCOLLAR). Maintenant, toutes ces étapes sont effectuées directement au Centre de Recherches géophysiques de Garchy. Ce qui fait que moins d'une journée après le vol, on peut obtenir les films traités. De plus, à tout moment du traitement, nous 




Fig. 1 : un radiomètre aéroporté. An air borne radiometer.

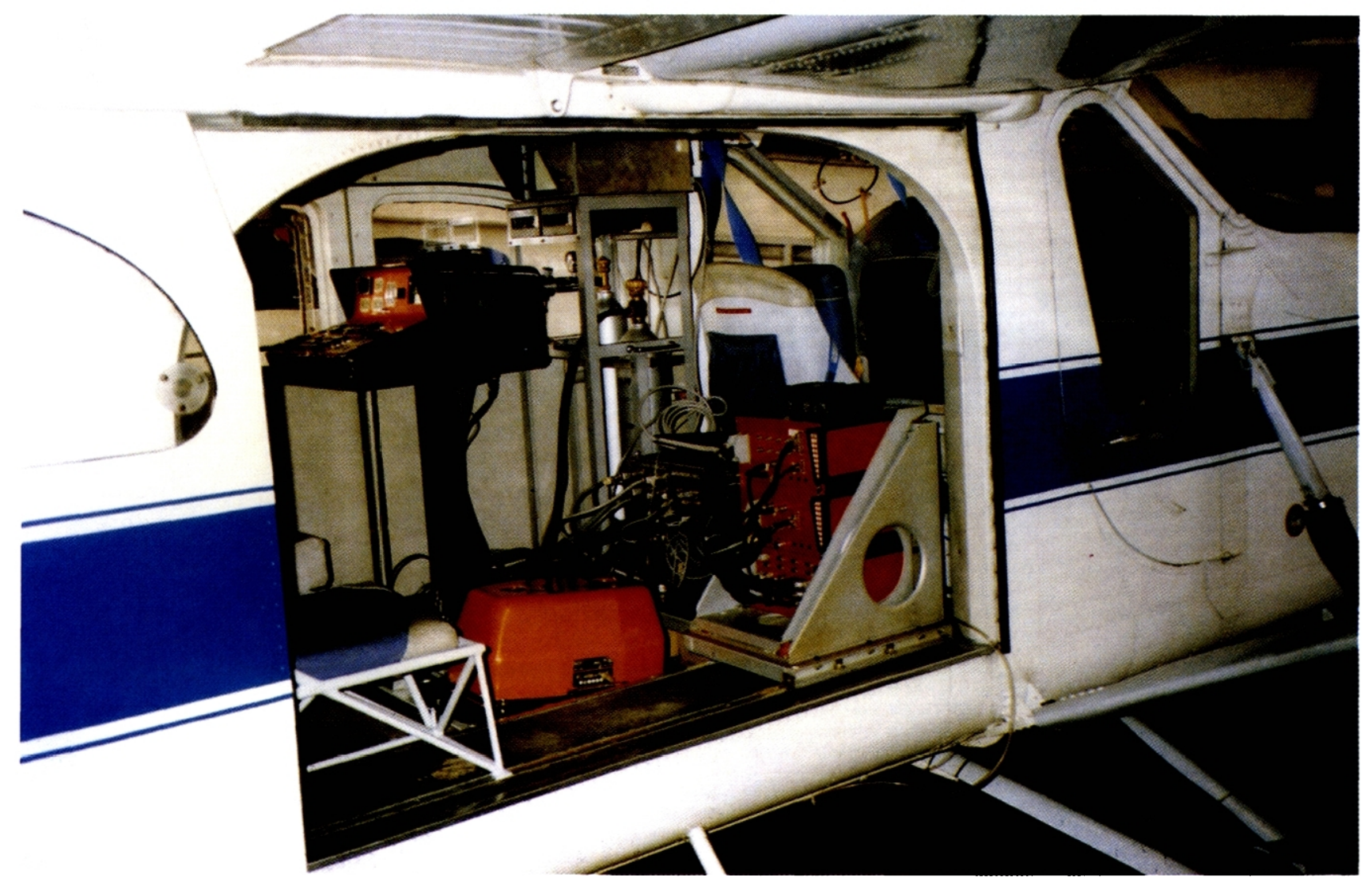

Fig. 2 : l'avion Pilatus et le matériel embarqué. Pilatus aircraft and on board apparatus. 


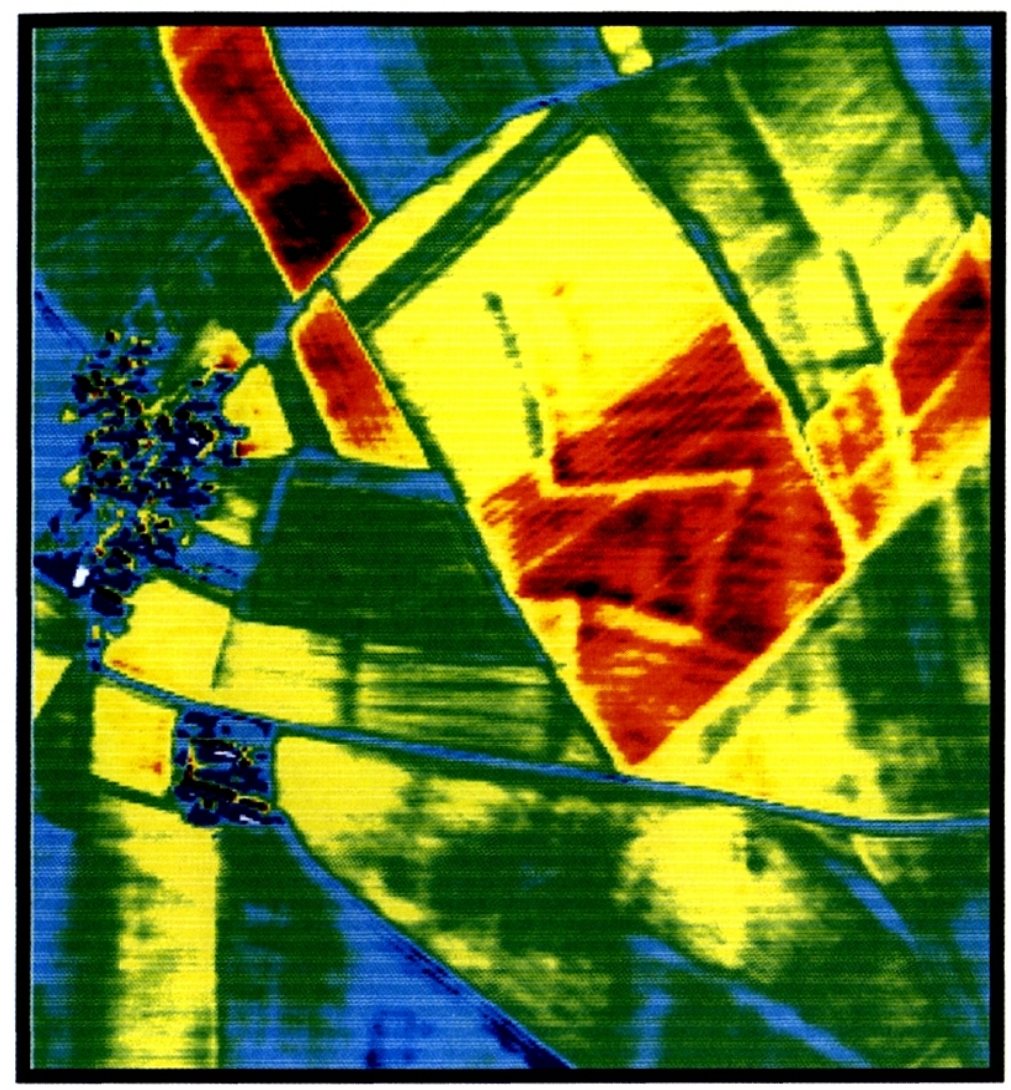

Fig. 3 : micro-reliefs en Beauce révélateurs d'un ancien parcellaire. Micro-relief surveyed in Beauce, originate from old field boundaries.

contrôlons la fiabilité des algorithmes sur les images affichées à l'écran.

\section{ARCHIVAGE}

Chaque mission est accompagnée d'un rapport avec la position topographique des profils et les paramètres de vol. Mais ce qui fait la force de notre système est l'enregistrement sur disque optique de tous les profils bruts et de tous les paramètres de vol de l'avion. Les noms de fichiers sont standardisés de façon à reconnaître le lieu de vol, l'année de la mission et le numéro de l'axe. Nous avons repris toutes nos archives (une centaine de bandes magnétiques depuis 1977) et les avons réinscrites sur disque optique avec la codification déjà mentionnée. Cinq disques optiques ont permis d'enregistrer les 1,5 giga-octets d'images, c'est-àdire près de 1600 kilomètres de profils.

Précisons que cette banque de donnée est interrogeable sur un PC ordinaire et les programmes sont conçus pour être utilisés par des néophytes. Des utilisateurs peuvent donc consulter cette base d'images.

Le choix du jour du vol (février-mars ou fin de l'automne pour bénéficier des sols nus), est défi- ni après des simulations numériques prenant en compte les données d'observatoires météorologiques au sol qui nous communiquent les températures jour après jour à différentes profondeurs. Grâce à ces données, nous calculons la réponse en température engendrée par des corps archéologiques et décidons du vol si les anomalies de température sont suffisamment élevées. Il est très important de voler le matin pour éviter l'élévation de température due au soleil qui masquerait les anomalies plus faibles.

\section{INTERPRÉTATION - UTILISATION}

Toutes les étapes antérieures, très automatisées, font très peu intervenir l'homme directement dans un schéma explicatif du paysage observé. La photographie aérienne oblique quant à elle implique, par opposition à nos relevés verticaux, un choix plus ou moins conscient du prospecteur aérien (choix de la zone photographiée correspondant à des anomalies détectées à l'œil nu, choix de l'angle de vue et de la position par rapport au soleil), choix plus ou moins subjectif.

Point si bien souligné par Roger Agache le premier jour de ce symposium quand il déclarait : " la tare la plus sérieuse de la photographie aérien- 
ne est la subjectivité ». Il avait peu après ajouté «l'œil ne peut tout enregistrer». Nous enregistrons avec la thermographie à la fois ce que l'œil ne peut pas voir par sa physiologie même, mais aussi nous enregistrons exhaustivement une telle quantité de données, que bien souvent, assis devant la console, de nouvelles structures apparaissent au fur et à mesure des traitements (fig. 3). Le travail de photointerprétation peut alors commencer dans un contexte général.

\section{BIBLIOGRAPHIE}

COLLECTIF (1992) - « Une cartographie de haut vol ", Journal du CNRS, n 33, Sept. 1992, p. 23.

SCOLLAR et al. (1990) - Archaeological prospecting and remote sensing. Cambridge, Univ. Press.

MONGE J.-L. et SIRIOU F. (1975) - ARIES : un radiomètre multispectral à balayage, Ve journée d'optique spatiale, Marseille. 